\title{
Processing unrelated language can change what you see
}

\author{
Alexia Toskos Dils and Lera Boroditsky \\ Stanford University, Stanford, California
}

\begin{abstract}
When we hear a story, do we naturally imagine the visual scene being described? Do the representations derived in the course of normal language comprehension interact with visual perception broadly? For example, might understanding language change how we interpret visual scenes, even when the visual scenes are unrelated to the linguistic content? In our study, people interpreted an ambiguous image after they had (1) seen real visual motion either upward or downward (Experiment 1), (2) read a story describing physical motion (Experiment 2), or (3) read a story describing abstract motion (Experiment 3). The ambiguous figure could have been seen as a bird flying upward or a different bird flying downward, and the participants were simply asked to click on or draw a worm in the bird's beak. People's interpretations of the ambiguous figure were affected by viewing real motion and by reading literal stories describing physical motion, but not by the abstract motion stories. These findings suggest that processing linguistic descriptions of physical (but not abstract) motion can bias perceptual processing in a broad sense; in this case, reading about physical motion changed people's interpretation of an unrelated ambiguous image.
\end{abstract}

When we hear a story, do we naturally imagine the visual scene being described? Do the representations derived in the course of normal language comprehension interact with visual perception broadly? For example, might processing linguistic information change how we attend to and interpret an unrelated visual scene?

Language is one domain in which mental imagery seems particularly useful, since people often talk about objects and events that are not in the immediate environment. Some researchers have suggested that language processing involves imagining or perceptually simulating the visual scenes being described, and have demonstrated that processing language can affect the speed and accuracy of processing visual stimuli (Barsalou, 1999; Bergen, Lindsay, Matlock, \& Narayanan, 2007; Estes, Verges, \& Barsalou, 2008; Gallese \& Lakoff, 2005; Glenberg \& Kaschak, 2002; Matlock, Ramscar, \& Boroditsky, 2005; Meteyard, Bahrami, \& Vigliocco, 2007; Richardson, Spivey, Barsalou, \& McRae, 2003; Spivey \& Geng, 2001; Stanfield \& Zwaan, 2001; Zwaan, Madden, Yaxley, \& Aveyard, 2004; Zwaan, Stanfield, \& Yaxley, 2002). For example, Stanfield and Zwaan showed that people are faster to respond to an image of a vertical nail following a sentence such as "Mary pounded the nail into the floor" than after "Mary pounded the nail into the wall" (and the reverse for an image of a horizontal nail). In this case, language processing seems to generate representations specific enough to affect visual processing of objects that are similar, in both structure and content, to those mentioned in the sentence.
Others have shown that the effects of language extend to perceptual tasks unrelated to the linguistic content. For example, Meteyard et al. (2007) found that processing directional motion verbs can impair people's accuracy in judging the motion coherence of a moving-dots display. Bergen et al. (2007) and Richardson et al. (2003) found that hearing sentences that direct attention upward ("The mule climbed," "The ceiling cracked") makes people slower to respond to an unrelated visual stimulus in the upper half of the visual field or along the vertical axis of fixation.

In this article we ask whether the representations derived in the course of normal language processing also interact with visual perception in a broader sense. Previous studies have focused on the speed or accuracy of responding to visual stimuli. Might processing linguistic information affect not just how quickly or accurately we can identify a visual stimulus but also what we actually interpret that visual stimulus to be? Could there be perceptual consequences even for interpreting visual stimuli that are unrelated to the linguistic content?

We approach these questions in the domain of motion. In our experiments, people were asked to interpret an ambiguous figure that could be seen as either a bird flying upward or a different bird flying downward (the ambiguous goose/hawk shown in Figure 1) under one of three conditions: after viewing real visual motion either upward or downward, after reading a story describing literal motion either upward or downward, or after reading a story describing abstract motion (where the things said

A. T. Dils, atoskos@psych.stanford.edu 

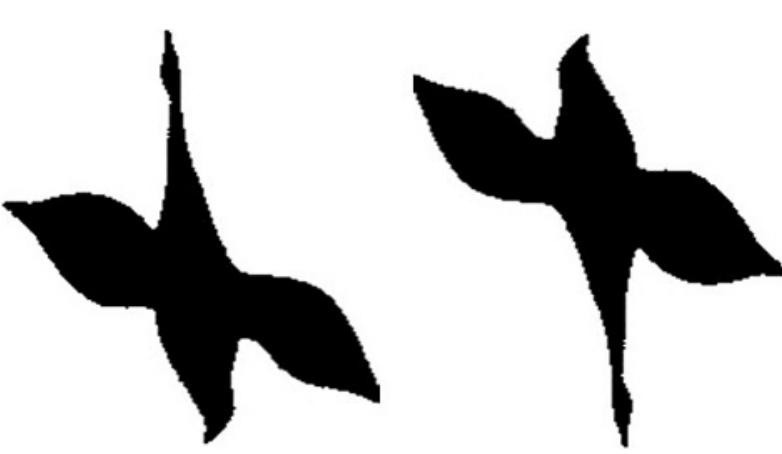

Figure 1. Ambiguous goose/hawk images.

to be moving are not physical entities and do not undergo translation in space-e.g., rising or falling stock prices). We first investigated whether viewing real motion affects how people interpret the ambiguous image, and then asked whether the same effects can be produced by reading about literal or abstract motion.

Previous work has demonstrated that induced apparent motion in a particular direction can bias participants to perceive a subsequent, ambiguously moving stimulus as moving in that same direction (Pantle, Gallogly, \& Piehler, 2000). Bernstein and Cooper (1997) examined the influence of induced, apparent, and smooth motion on people's interpretation of the facing direction of the ambiguous goose/hawk figure we used in our study. They found that both induced and apparent motion draw people's attention in the direction of motion, and this attentional shift biases the perceived facing direction of the bird. These results predict that an ambiguous perceptual stimulus presented just after viewing motion in a single direction (which would draw a shift in spatial attention in that direction) should be disambiguated as facing that same direction.

\section{EXPERIMENT 1 Real Visual Motion}

\section{Method}

In Experiment 1, participants first watched a video depicting either upward or downward visual motion and were then asked to interpret an ambiguous image.

Participants. Ninety-seven workers from Amazon's Mechanical Turk Web site participated for $\$ 0.35$ pay.

Materials. The motion stimuli were two videos showing moving sine gratings, one moving upward and one moving downward at a rate of 3 cycles per second. Effects of the motion stimuli were measured using an ambiguous figure centered on the screen. The image could be interpreted as either a hawk facing one direction or a goose facing the opposite direction (Tinbergen, 1951). Both orientations of the ambiguous figure are shown in Figure 1. The moving grating was smaller in both width and height than the bird image (the grating:bird minimal rectangle area ratio was $0.7: 1$ ).

Procedure. Participants completed the task online, and all task materials were presented in an interactive Adobe Flash video. Participants first watched a 30-sec video of either upward or downward moving stripes (having been instructed to fixate and also keep their mouse cursor on the central fixation cross superimposed on the video). Immediately afterward, the ambiguous goose/hawk image appeared on the screen, and participants were asked to "click on the bird's beak." This was done to avoid explicitly asking participants about their interpretation of the ambiguous figure and to not draw attention to the ambiguity. We recorded the $x$ - and $y$-coordinates of the mouse-click; clicks above fixation were coded as an "upward" interpretation, and clicks below fixation were coded as a "downward" interpretation. The orientation of the ambiguous image was counterbalanced across participants and across motion types.

Finally, participants were asked to describe anything "interesting" that they noticed about the image. The purpose of this question was to identify participants who noticed the ambiguity.

\section{Results}

Twelve participants indicated that they noticed that the bird image had two possible interpretations and were excluded from further analysis. There was an overall bias for participants to see the ambiguous figure as a goose $(64 \%)$ rather than a hawk $(36 \%)\left[\chi^{2}(1, N=85)=6.22\right.$, $p<.05]$. Overall, participants were equally likely to see the figure as an upward-facing bird or a downward-facing bird. Slightly more participants (51\%) made the downward interpretation than the upward interpretation (49\%), but this difference was not reliable $\left[\chi^{2}(1, N=85)=0.01\right.$, $p>.90]$.

Importantly, participants' interpretations of the ambiguous bird image were influenced by the direction of the motion they had just seen. Participants were significantly more likely to see a bird facing in the same direction as the motion in the video $(61 \%)$ than in the opposite direction $(39 \%)\left[\chi^{2}(1, N=85)=4.25, p<.05\right]$. Results for all three experiments are plotted in Figure 2.

\section{Discussion}

The results of Experiment 1 show a perceptual congruity effect: Participants were more likely to perceive the ambiguous image as a bird facing in the same direction as the motion in the video they had just seen than as a different bird facing in the opposite direction. These findings are consistent with those of Bernstein and Cooper (1997). It seems that drawing visuospatial attention in a particular direction biases interpretation of the ambiguous figure as facing in that same direction. In Experiment 2, we asked whether reading a passage describing motion in a particular direction (instead of watching real motion) could have the same effect on subsequent perceptual processing.

\section{EXPERIMENT 2 Literal Motion}

\section{Method}

Participants. Four hundred sixty-five undergraduates from Stanford University and the University of California, Merced participated for course credit.

Materials. The two paragraphs we used, one describing upward and one describing downward motion, are shown in Table 1. Neither directional prepositions nor directional verbs were used to establish upward or downward motion. Rather, the directionality of the story emerged at the sentence level.

Procedure. Participants completed the task on paper as part of a large questionnaire packet that contained many unrelated tasks. Each participant read either the upward or the downward version of the story, followed by a comprehension question: "Do the children collect any prizes before the final floor?" This question was included to make sure participants read the story. On the next page, participants saw the ambiguous goose/hawk figure (centered on the page) 


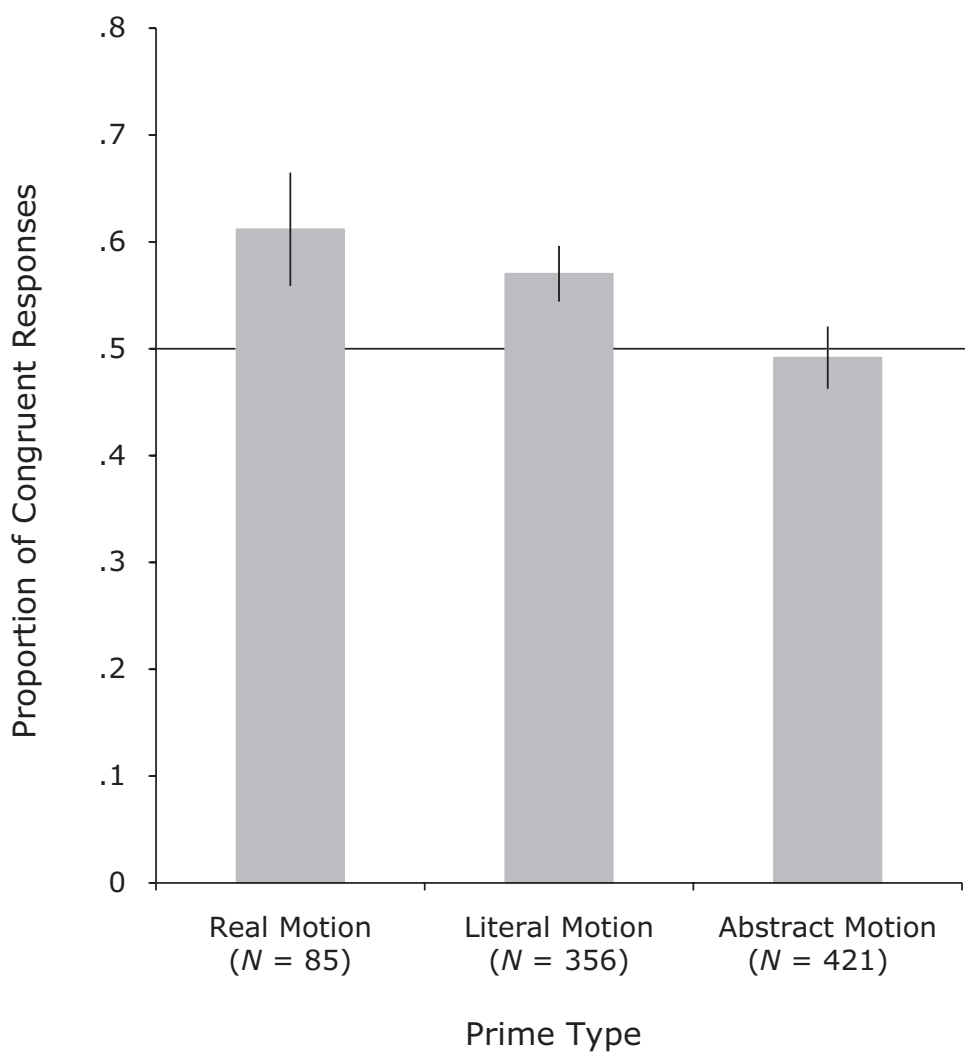

Figure 2. Proportions of congruent interpretations of the ambiguous goose/ hawk image following real visual motion (Experiment 1), literal linguistic motion (Experiment 2), and abstract linguistic motion (Experiment 3). Error bars represent the standard error of the proportion, and the horizontal line reflects chance performance.

and were asked to "draw a worm in the bird's beak." As before, the orientation of the ambiguous image was counterbalanced across participants and across motion types. Participants who drew the worm at the top of the image were coded as having made an "upward" interpretation of the image, and participants who drew the worm at the bottom of the image were coded as having made a "downward" interpretation of the image. Because the questionnaire packet contained many unrelated tasks, all on separate pages, participants may not have had an a priori reason to believe that the two pages of the questionnaire comprising our study were related.

\section{Results}

Eighty-seven (out of 465) participants either omitted or incorrectly answered the comprehension question and were excluded from all analyses. Of those remaining, $22^{1}$ participants noticed that the bird image had two possible interpretations (i.e., drew worms in both beaks) and were also excluded.

Overall, participants were equally likely to interpret the image as a goose or a hawk. Slightly more participants $(52 \%)$ made the goose interpretation than the hawk interpretation (48\%), but this difference was not reliable $\left[\chi^{2}(1, N=356)=0.55, p>.45\right]$. There was an overall bias for participants to see a downward-facing bird (57\%) rather than an upward-facing bird $(43 \%)\left[\chi^{2}(1, N=\right.$ $356)=7.60, p<.01]$.
Importantly, participants' interpretation of the ambiguous bird image was influenced by the direction of the motion in the story they had just read. Participants were significantly more likely to see a bird facing in the same direction as the motion in the story (57\%) than in the opposite direction (43\%) $\left[\chi^{2}(1, N=356)=7.02, p<\right.$ $.01]$. The pattern of results found in Experiment 2 does not differ reliably from the pattern found in Experiment 1 $\left[\chi^{2}(1, N=441)=0.49, p>.45\right]$.

\section{Discussion}

Participants were more likely to perceive the ambiguous image as a bird facing in the same direction as the direction of the motion in the story they had just read than as a different bird facing in the opposite direction. This bias was indistinguishable from that produced by viewing real visual motion. Both hearing stories describing spatial motion (Experiment 2) and viewing real motion (Experiment 1) (as well as induced, apparent, and smooth motion in Bernstein \& Cooper, 1997) appear to draw people's attention in the direction of motion such that they interpret the facing direction of the bird as congruent with the direction of the motion they had just seen or heard about.

In Experiment 3, we extended this paradigm to investigate whether abstract or metaphorical descriptions of mo- 
Table 1

Stories Read by Participants in Experiment 2

Upward Motion Story

You are standing on the 29th floor of an artsy 57-story building looking into a beautiful atrium. You notice a group of third-grade children beginning a treasure hunt on the first floor. They pile into the giant glass elevator across from you with the first clue in hand. You watch as they solve the first clue, which sends them to the 9th floor for the next one. From there, they have to rush to the 20th floor. From the 20th floor, they follow the clue to the 29 th floor. They solve the clue on the 29th floor and then have to hurry to the 38 th floor. From the 38 th floor, the clue sends them to the 49 th floor. The final clue sends them from the 49th floor all the way to the 57 th floor, where they excitedly collect their prize!

\section{Downward Motion Story}

You are standing on the 29th floor of an artsy 57-story building looking into a beautiful atrium. You notice a group of third-grade children beginning a treasure hunt on the first floor. They pile into the giant glass elevator across from you with the first clue in hand. You watch as they solve the first clue, which sends them to the 49th floor for the next one. From there, they have to rush to the 38th floor. From the 38th floor, they follow the clue to the 29th floor. They solve the clue on the 29th floor and then have to hurry to the 20th floor. From the 20th floor, the clue sends them to the 9th floor. The final clue sends them from the 9 th floor all the way to the first floor, where they excitedly collect their prize!

Comprehension question: Do the children collect any prizes before the final floor?

tion likewise have perceptual consequences. Metaphorical language poses a further interesting case for the investigation of mental simulation. What representations do people form when they hear things such as "The prices soared" or "the economy went into a nosedive"? We often talk about abstract entities (in this case, prices and the economy) metaphorically. Do people create perceptual representations when they hear that "the economy went into a nosedive" that are similar to those for "the bomber went into a nose-dive"? Some previous studies have shown evidence of perceptual consequences of abstract language processing (Richardson et al., 2003), whereas others have not (e.g., Bergen et al., 2007; Boroditsky, 2000; see Bergen et al., 2007, for a discussion of possible reasons for this discrepancy). To investigate this question in our paradigm, we replaced the literal motion stories used in Experiment 2 with stories describing abstract motion.

\section{EXPERIMENT 3 Abstract Motion}

In Experiment 3, participants read a story that described either upward or downward abstract motion and then interpreted the ambiguous goose/hawk image. The stories included the same rising and falling numbers as used in the elevator stories in Experiment 2, but, instead of describing physical motion, the numbers described changing stock prices or changing championship rankings. An early pilot of this task did not produce any effect of the linguistic prime. Out of concern that the abstract stories may have been less vivid or engaging than the literal stories, we tested several variations of the stories using more vivid language in two versions.

\section{Method}

Participants. We tested 558 participants in total. Participants included 348 undergraduates from Foothill College and the University of California, Merced (for course credit) and 210 workers from Amazon's Mechanical Turk Web site (for $\$ 0.35$ pay).

Materials. The passages used (and the corresponding comprehension questions) are shown in Table 2. Two hundred sixteen participants were tested on Version 1 of the abstract stories (as seen in Table 2). These stories contained no directional verbs and were designed to closely mirror the literal stories from Experiment 2 . Two hundred eighty-eight participants were tested on a slightly different version (Version 2), in which the stock prices "plummeted" or "skyrocketed." These stories were designed with additional cues to the depicted motion trajectory, with the direction of motion encoded at both the verb and sentence levels. Fifty-four participants were tested on Version 3 stories, which described rising or falling championship rankings, in which low rankings were positive and high rankings were negative. The stories in Version 3 were designed to decorrelate the spatial information provided by the verbs and numbers in Version 2 and to de-correlate motion direction and valence. The procedure for Experiment 3 was identical to that for Experiment 2 for participants filling out the paper-and-pencil version of the experiment and was modeled on Experiment 1 for online participants. All online participants were run on Version 2 stories.

\section{Results}

Sixty-one (out of 558) participants either omitted or incorrectly answered the comprehension question and were excluded from all analyses. Seventy-six participants noticed that the bird image had two possible interpretations (i.e., drew worms in both beaks) and were also excluded.

There was an overall bias for participants to see the ambiguous figure as a goose (61\%) rather than a hawk $(39 \%)$ $\left[\chi^{2}(1, N=421)=18.82, p<.001\right]$ and a bias for participants to see a downward-facing bird (58\%) rather than an upward-facing bird $(42 \%)\left[\chi^{2}(1, N=421)=10.66\right.$, $p<.005]$.

Importantly, participants' interpretation of the ambiguous goose/hawk image was not influenced by the direction of the abstract motion in the story they had just read. Participants were slightly less likely to see a bird facing in the same direction as the motion in the story (49\%) than one facing in the opposite direction (51\%), but this difference was not reliable $\left[\chi^{2}(1, N=421)=0.12, p>.70\right]$. The pattern of results did not significantly differ across the three versions of Experiment 3 (Versions 1, 2, and 3 yielded $48 \%, 51 \%$, and $44 \%$ congruent responses, respectively, none of which differ reliably from chance (all $p \mathrm{~s}>$ .45) $\left[\chi^{2}(2, N=421)=0.72, p>.65\right]$, so the data from all abstract motion stories were combined for all analyses. Importantly, the pattern of results found in Experiment 3 differs reliably from the patterns found in both Experiment $1\left[\chi^{2}(1, N=506)=4.08, p<05\right]$ and Experiment 2 $\left[\chi^{2}(1, N=777)=4.77, p<.05\right]$.

The results of Experiment 3 show no bias in participants' interpretation of the ambiguous stimulus as a function of reading about abstract motion in a particular direction. 
Table 2

Stories Read by Participants in Experiment 3

Version 1: Upward Motion Story

It is your company's first day on the stock market and you are watching carefully to see what happens with the price. The stock opens at $\$ 1$ a share. Before you know it, the price goes to $\$ 9$. From there the price goes to $\$ 20$. From $\$ 20$, the stock rushes to $\$ 29$. From $\$ 29$, the stock goes to $\$ 38$. From $\$ 38$, the price rushes to $\$ 49$. Finally, the price goes all the way to $\$ 57$.

\section{Version 1: Downward Motion Story}

It is your company's first day on the stock market and you are watching carefully to see what happens with the price. The stock opens at $\$ 57$ a share. Before you know it, the price goes to $\$ 49$. From there the price goes to $\$ 38$. From $\$ 38$, the stock rushes to $\$ 29$. From $\$ 29$, the stock goes to $\$ 20$. From $\$ 20$, the price rushes to $\$ 9$. Finally, the price goes all the way to $\$ 1$.

Comprehension question: In the story you just read, was it your company's first day on the market?

Version 2: Upward Motion Story

It is your company's first day on the stock market and you are watching carefully to see what happens with the price. The stock opens at $\$ 1$ a share. Before you know it, the price jumps to $\$ 9$. From there the price shoots to $\$ 20$. From $\$ 20$, the stock zooms to $\$ 29$. From $\$ 29$, the stock jumps to $\$ 38$. From $\$ 38$, the price shoots to $\$ 49$. Finally, the price skyrockets all the way to $\$ 57$.
Version 2: Downward Motion Story

It is your company's first day on the stock market and you are watching carefully to see what happens with the price. The stock opens at $\$ 57$ a share. Before you know it, the price drops to $\$ 49$. From there the price dives to $\$ 38$. From $\$ 38$, the stock plunges to $\$ 29$. From $\$ 29$, the stock drops to $\$ 20$. From $\$ 20$, the price dives to $\$ 9$. Finally, the price plummets all the way to $\$ 1$.

Comprehension question: In the story you just read, was it your company's first day on the market?

Version 3: Upward Motion Story

It is your team's first championship and you are watching carefully to see what happens with the rankings. The team starts the championship rated \#1. Before you know it, the ranking drops to \#9. From there the ranking dives to $\# 20$. From $\# 20$, the ranking plunges to $\# 29$. From \#29, the team drops to \#38. From \#38, the ranking dives to \#49. Finally, the ranking plummets all the way to \#57.
Version 3: Downward Motion Story

It is your team's first championship and you are watching carefully to see what happens with the rankings. The team starts the championship rated \#57. Before you know it, the ranking jumps to \#49. From there the ranking shoots to \#38. From \#38, the ranking zooms to $\# 29$. From $\# 29$, the team jumps to \#20. From \#20, the ranking shoots to \#9. Finally, the ranking skyrockets all the way to \#1.

Comprehension question: In the story you just read, was it your team's first championship?

\section{GENERAL DISCUSSION}

Together, the three experiments suggest that processing descriptions of physical but not abstract motion has perceptual consequences for subsequent, unrelated visual tasks. The effects of processing literal motion are indistinguishable in this task from those produced by viewing real visual motion. These findings are also consistent with disambiguation patterns caused by induced, apparent, and smooth motion reported in earlier studies and lend support to the attentional shift mechanisms postulated to explain such effects (Bernstein \& Cooper, 1997). When it comes to abstract motion descriptions, however, we found no evidence of perceptual consequences. One important way in which our results extend previous work is by showing that processing language not only can affect how quickly or accurately people can respond to a visual stimulus, but also can cause a qualitative shift in what people actually interpret the stimulus to be. This is true even when the visually relevant information (in this case, the direction of motion) is not encoded in individual lexical items but, instead, must be extracted at the paragraph level, and even though the visual stimulus to be interpreted is unrelated to the content of the language being processed. It seems that representations created in the course of understanding language interact rather broadly with visual processing.

These findings raise a number of interesting questions about the nature of the representations people create in the process of normal language comprehension. Why would reading a story about an elevator moving up and down in a building influence how people interpret an unrelated ambiguous image of a bird? One possibility is that people create a dynamic mental image of the perceptual scene described in the story. If the mental image is similar enough to the experience of real visual motion (if it directs visual attention in the same way, for example), then it can have the same perceptual consequences as does real visual motion. This interpretation is consistent with our results.

However, another possibility is that the congruity or priming effect observed in Experiment 2 does not rely on recruiting visual attention mechanisms in the course of language processing at all. It could be that when people read a story about an elevator going up, they generate an abstract symbolic representation of $u p$, and it is this abstract representation that then biases their interpretation of the ambiguous image. This abstract representation of up could be invoked simply by the rising floor numbers, for example, without any dynamic mental images, shifts in visual attention, or even representation of motion necessary.

The results of Experiment 3 can help us disambiguate these two possibilities. The stories in Experiment 3 contained the same rising and falling numbers (for stock prices) as did the stories in Experiment 2 (for floors). If rising or falling numbers were sufficient to generate the congruity effect we observed in Experiment 2, then we should have seen the same effect in Experiment 3. Furthermore, the stories used in Experiment 3 (Version 2) very clearly invoked the notions of $u p$ and down with vivid directional verbs such as skyrocket and plummet. If simply priming a notion of $u p$ or down was enough to generate the congruity effects in Experiment 2, then we should have seen similar effects in Experiment 3. That no such congruity effects were found in Experiment 3 suggests that 
neither rising and falling numbers nor simple priming of an abstract notion of up or down can be the explanation for the effects we observe in Experiment 2.

A further question is why indeed the abstract motion stories in Experiment 3 failed to produce the perceptual congruency effect found with the literal motion stories in Experiment 2. It is possible that participants simply did not create visual images to represent the contents of these stories, or perhaps that the visual images they created lacked some key components. The images people created may not have been vivid enough or may not have been dynamic. Another possibility is that what people imagined was the basic (and likely stationary) physical environment suggested by the stories (e.g., sitting in front of a computer monitor, looking at a display board with numbers), rather than the abstract entities themselves. If this is the case, then the visual images of the stationary physical environment may have preempted visualizing abstract motion and shifting attention in a particular direction. One way to test for this possibility would be to prevent participants from visualizing themselves in a physical environment by removing them from the story (our stories were written in the second person and so would have invited the participants to imagine themselves at the center of the action). Another approach would be to make the physical environment suggested by the story invisible (e.g., "You are sitting in complete darkness and listening to stock reports").

As they stand, our findings are consistent with the existing body of work on the perceptual consequences of metaphorical or abstract language processing (e.g., Bergen et al., 2007; Boroditsky, 2000). For example, Boroditsky found that although processing literal spatial language influenced the interpretation of an ambiguous visual image, processing similarly structured metaphorical spatiotemporal language did not. Importantly, these studies do not show that metaphorical or abstract representations are unrelated to spatial or perceptual representations but, rather, show that their relationship is asymmetrical. Priming a set of concrete spatial representations affected how people processed spatiotemporal metaphors (Boroditsky, 2000; Boroditsky \& Ramscar, 2002), but priming spatiotemporal metaphors did not influence how people reasoned about space. The results of the present study are consistent with these findings. It seems that our perceptual representations may be less susceptible to influence from representations of the abstract than the other way around.

\section{CONCLUSIONS}

Viewing real visual motion and reading descriptions of physical motion (but not descriptions of abstract motion) affected the way in which participants perceived an unrelated visual image. Specifically, participants showed a consistent bias toward interpreting an ambiguous visual image as a bird facing in the same direction as the motion described in an unrelated story, rather than as a different bird facing in the opposite direction. This congruity effect suggests that there is overlap between the representa- tions and/or attention processes involved in understanding language and in interpreting visual images. It seems that representations created as a result of processing language can have broad effects on perception. In this case, processing unrelated language can not only change how quickly or accurately you can respond to a visual stimulus, but also qualitatively change what you interpret the stimulus to be.

\section{AUTHOR NOTE}

Many thanks to Jonathan Winawer, Caitlin Fausey, and the rest of Cognation for helpful discussions regarding this work, and to Teenie Matlock for making data collection at UC Merced possible. This research was funded by NSF Grant 0608514 to L.B. Correspondence concerning this article should be addressed to A. T. Dils, Department of Psychology, Stanford University, 450 Serra Mall, Bldg. 420, Stanford, CA 94304 (e-mail: atoskos@psych.stanford.edu).

\section{Note-Accepted by Cathleen M. Moore's editorial team.}

\section{REFERENCES}

Barsalou, L. W. (1999). Perceptual symbol systems. Behavioral \& Brain Sciences, 22, 577-660. doi:10.1017/S0140525X99002149

Bergen, B. K., Lindsay, S., Matlock, T., \& Narayanan, S. (2007). Spatial and linguistic aspects of visual imagery in sentence comprehension. Cognitive Science, 31, 733-764. doi:10.1080/03640210701530748

Bernstein, L. J., \& Cooper, L. A. (1997). Direction of motion influences perceptual identification of ambiguous figures. Journal of Experimental Psychology: Human Perception \& Performance, 23, 721737. doi:10.1037/0096-1523.23.3.721

Boroditsky, L. (2000). Metaphoric structuring: Understanding time through spatial metaphors. Cognition, 75, 1-28. doi:10.1016/S0010 -0277(99)00073-6

Boroditsky, L., \& RAMSCAR, M. (2002). The roles of the body and mind in abstract thought. Psychological Science, 13, 185-188. doi:10.1111/ $1467-9280.00434$

Estes, Z., Verges, M., \& Barsalou, L. W. (2008). Head up, foot down: Object words orient attention to the object's typical location. Psychological Science, 19, 93-97. doi:10.1111/j.1467-9280.2008.02051.x

Gallese, V., \& LAKOFF, G. (2005). The brain's concepts: The role of the sensory-motor system in conceptual knowledge. Cognitive Neuropsychology, 22, 455-479. doi:10.1080/02643290442000310

GLENBERG, A. M., \& KaschaK, M. P. (2002). Grounding language in action. Psychonomic Bulletin \& Review, 9, 558-565.

Mast, F. W., \& Kosslyn, S. M. (2002). Visual mental images can be ambiguous: Insights from individual differences in spatial transformation abilities. Cognition, 86, 57-70. doi:10.1016/S0010-0277(02)00137-3

MatLock, T., Ramscar, M., \& Boroditsky, L. (2005). On the experiential link between spatial and temporal language. Cognitive Science, 29, 655-664. doi:10.1207/s15516709 $\operatorname{cog} 0000 \_17$

Meteyard, L., Bahrami, B., \& Vigliocco, G. (2007). Motion detection and motion verbs: Language affects low-level visual perception. Psychological Science, 18, 1007-1013. doi:10.1111/j.1467-9280.2007 .02016.x

Pantle, A. J., Gallogly, D. P., \& Piehler, O. C. (2000). Direction biasing by brief apparent motion stimuli. Vision Research, 40, 19791991. doi:10.1016/S0042-6989(00)00071-7

Richardson, D. C., Spivey, M. J., Barsalou, L. W., \& McRae, K. (2003). Spatial representations activated during real-time comprehension of verbs. Cognitive Science, 27, 767-780. doi:10.1016/S0364 $-0213(03) 00064-8$

Spivey, M., \& GenG, J. (2001). Oculomotor mechanisms activated by imagery and memory: Eye movements to absent objects. Psychological Research, 65, 235-241. doi:10.1007/s004260100059

Stanfield, R., \& ZWAan, R. (2001). The effect of implied orientation derived from verbal context on picture recognition. Psychological Science, 12, 153-156. doi:10.1111/1467-9280.00326

Tinbergen, N. (1951). The study of instinct. Oxford: Oxford University Press.

ZwaAn, R. A., Madden, C. J., YaXley, R. H., \& Aveyard, M. E. (2004). 
Moving words: Dynamic representations in language comprehension. Cognitive Science, 28, 611-619. doi:10.1016/j.cogsci.2004.03.004

Zwaan, R. A., Stanfield, R. A., \& Yaxley, R. H. (2002). Language comprehenders mentally represent the shapes of objects. Psychological Science, 13, 168-171. doi:10.1111/1467-9280.00430

\section{NOTE}

1. More participants noticed the ambiguity in the goose/hawk stimulus in Experiment $1(12 \%)$ than in Experiment $2(6 \%)$. It is possible that by not explicitly asking participants whether they noticed the ambiguity in Experiment 2, we underestimate the number of people who really did notice by $6 \%$. Let us assume, conservatively, that an additional $6 \%$ of participants in Experiment 2 noticed the ambiguity but drew only one worm, and that all of those participants drew the worm so that the bird was fac- ing in the same direction as the motion in the story. After removing those participants, the data would still go in the same direction, with $54 \%$ of participants seeing a bird facing in the same direction as the motion in the story. Another possibility is that by explicitly asking participants whether they noticed the ambiguity in Experiment 1 , we invite them to reinspect the goose/hawk image in memory (Mast \& Kosslyn, 2002) or otherwise guess what might have been interesting about the image after the fact. On this second possibility, we overestimate the number of participants in Experiment 1 who noticed the ambiguity at the time of stimulus presentation. Adding the $12 \%$ of participants in Experiment 1 who reported noticing the ambiguity still results in $60 \%$ of participants seeing a bird facing in the same direction as the motion in the video.

(Manuscript received March 9, 2010; revision accepted for publication July 28, 2010.) 